\title{
The Uplink Capacity of SA-MIMO System under Single-User Scenario
}

\author{
Jianxin Dai ${ }^{1,2}$, Ming Chen ${ }^{1}$, Member, IEEE \\ ${ }^{1}$ National Mobile Communications Research Lab., \\ Southeast University, Nanjing, 20096, China \\ ${ }^{2}$ School of Science, Nanjing University of Posts and \\ Telecommunications \\ daijx@nupt.edu.cn, chenming@seu.edu.cn
}

\begin{abstract}
The uplink capacity of the single-user smart antennas (SA) - multiple-input-multiple-output (MIMO) wireless systems, which combines smart antennas with MIMO at the receiver, is investigated in this paper. The joint optimization problem corresponding to the capacity is deduced, and in the case of equal power allocation and the same direction-of-arrivals (DOAs) from different transmit antennas at the same antenna array the closedform expression of the capacity is obtained, an upper bound of the capacity is also given in the case of different DOAs at the same antenna array. Some numerical results are also given to compare the capacities of conventional MIMO and SA-MIMO systems.
\end{abstract} forming

Keywords-smart antenna; MIMO; capacity; uplink; beam-

\section{INTRODUCTION}

As well known, MIMO system with multiple antennas at both the transmitter and receiver can increase significantly the capacity of wireless channel without requiring additional power or bandwidth [1-4], and smart antennas can suppress the interference coming from different directions by beam-forming and hence can make the cell has a wider coverage and greater user capacity [5-6]. Therefore, if combine MIMO and smart antennas, both advantages can be obtained. In this paper, we investigate the capacity of SA-MIMO system which is a combination of MIMO and smart antennas by replacing each antenna of MIMO with a smart antenna array.

For conventional MIMO system, the capacity can be achieved by power allocation in waterfilling manner when the transmitter has the complete channel state information (CSI)[1]. While for SA-MIMO system, the capacity will be achieved by jointly choosing the optimal beam-forming vectors and the power allocation scheme among the transmit antennas, so to give the closed expression form for the capacity of SA-MIMO system is more difficult than that of conventional MIMO system. In [7], a structure of MIMO system incorporated with smart antennas was proposed to reduce spatial correlation, and thus raised the MIMO channel capacity significantly. The two methods of combining beamforming with space-time block coding respectively using single antenna array and two dependent antenna arrays were proposed to get the full diversity order as well as beamforming gain in [8].

\author{
Pei-Jung Chung, Senior Member, IEEE \\ Institute for Digital Communications, \\ School of Engineering, the University of Edinburgh \\ Mayfield Road, Edinburgh EH9 3JL,UK \\ p.chung@ed.ac.uk
}

In the paper the optimization problem corresponding the capacity of uplink SA-MIMO system is deduced, and results are obtained in some cases.

The paper is organized as follows. In Section II, we introduce the single-user uplink SA-MIMO system model. Section III formulates the optimization problem. Section IV shows some numerical results. Finally, we make some concluding remarks in Section V.

\section{SYSTEM MODEL}

Consider an uplink SA-MIMO system which is shown in Fig. 1. The user transmitter has $N$ antenna transmitting uplink signals, their mutual distances are larger than the half wavelength of the carrier wave such that all the transmit channels are independent each other. At the receiver base station, there are $M$ antenna arrays, each of which has $L$ elements whose mutual distance is less than the half wavelength. In addition, all the $M$ antenna arrays are so far mutually that their receive channels are independent each other.

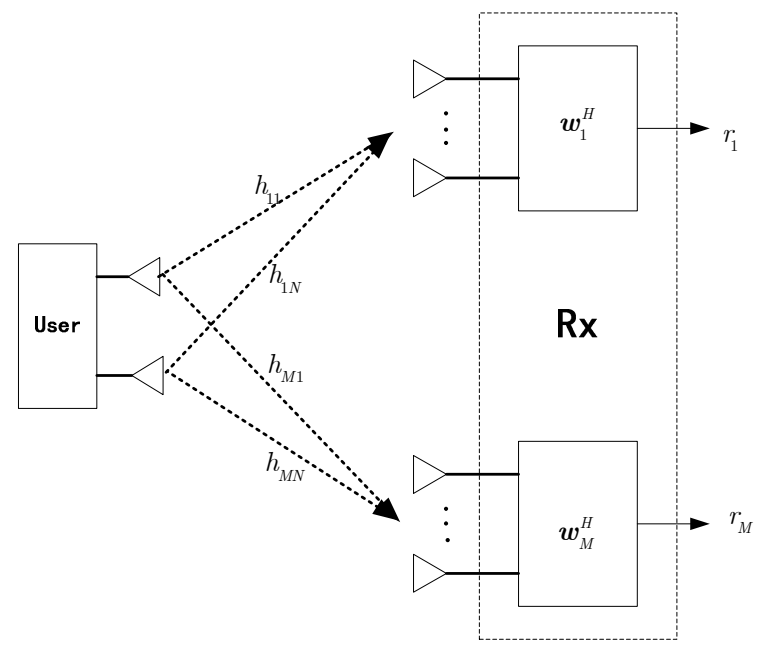

Fig. 1 Single-User uplink SA-MIMO System Model

Let $h_{m n}$ be the microscope fading coefficient between the $n$-th transmit antenna and the $m$-th receive antenna array for all $1 \leq m \leq M$ and all $1 \leq n \leq N$. The channels are flat Rayleigh fading, i.e., $h_{m n} \sim C N(0,1)$, where $x \sim C N\left(\mu, \sigma^{2}\right)$ 
means that $x$ is complex Gaussian distributions with mean $\mu$ and variance $\sigma^{2}$.

Denote the beam-forming vector of the $m$-th array at the receiver as $\boldsymbol{\omega}_{m}=\left(\omega_{m, 1}, \omega_{m, 2}, \cdots, \omega_{m, L}\right)$, and $\left\|\boldsymbol{\omega}_{m}\right\|=1$, where $\|\bullet\|$ is the Euclid norm of a vector, and let $\boldsymbol{a}_{m n}=\boldsymbol{a}\left(\theta_{m n}\right)$ be the steering vector of the $m$-th antenna array with respect to the $n$-th transmit antenna, and $s_{1}, s_{2}, \cdots, s_{N}$ and $p_{1}, p_{2}, \cdots, p_{N}$ be the transmitted signals and powers at the $N$ antennas, then the received signal at the $m$-th antenna array after beam-forming can be written as

$$
\begin{aligned}
r_{m} & =\sum_{n=1}^{N} s_{n} \sqrt{p_{n}} h_{m n}\left\langle\boldsymbol{\omega}_{m}, \boldsymbol{a}_{m n}\right\rangle+\left\langle\boldsymbol{\omega}_{m}, \boldsymbol{z}_{m}\right\rangle \\
& \triangleq \sum_{n=1}^{N} s_{n} \sqrt{p_{n}} \tilde{h}_{m n}+\tilde{z}_{m}
\end{aligned}
$$

where $\boldsymbol{z}_{m}=\left(z_{m, 1}, \cdots, z_{m, L}\right) \sim C N\left(\boldsymbol{O}, \sigma^{2} \boldsymbol{I}_{L}\right)$ is the noise vector at the $m$-th antenna array, $\langle$,$\rangle denotes the Euclid inner$ product of two vectors .

By stacking the received signals of all antenna arrays into $\mathbf{r}=\left(r_{1}, \cdots, r_{N}\right)^{\mathrm{T}}$, we have

$$
\boldsymbol{r}=\boldsymbol{H P} s+z
$$

where $\boldsymbol{z}=\left(\tilde{z}_{1}, \cdots, \tilde{z}_{M}\right)^{\mathrm{T}}, \boldsymbol{s}=\left(s_{1}, \cdots, s_{N}\right)^{\mathrm{T}}, \boldsymbol{H}=\left(\tilde{h}_{m n}\right)_{M \times N}$,

$$
\boldsymbol{P}=\operatorname{diag}\left[\sqrt{p_{1}}, \cdots, \sqrt{p_{N}}\right] \text {. }
$$

\section{INFORMATION THEORETIC CAPACITY}

Let $\mathbb{W}=\left\{\boldsymbol{\omega}_{1}, \boldsymbol{\omega}_{2}, \cdots, \boldsymbol{\omega}_{M}\right\}$. Assuming a circularly symmetric complex gaussian i.i.d. input signal vector $s$ with covariance function $\mathbb{E}\left\{s s^{\mathrm{T}}\right\}=\boldsymbol{I}_{N}$, the instantaneous capacity of the SAMIMO system for each channel use can be formulated as:

$$
\begin{gathered}
C=\max _{\mathbb{W}, \boldsymbol{P}}\left\{\log _{2} \operatorname{det}\left(\boldsymbol{I}_{M}+\frac{1}{\sigma^{2}} \boldsymbol{H} \boldsymbol{P} \boldsymbol{P}^{\mathrm{H}} \boldsymbol{H}^{\mathrm{H}}\right)\right\} \\
\text { s.t. } \\
\left\|\boldsymbol{\omega}_{m}\right\|=1, \quad \forall \boldsymbol{\omega}_{m} \in \mathbb{W} \\
\operatorname{Tr}\left(\boldsymbol{P} \boldsymbol{P}^{\mathrm{H}}\right)=\sum_{n=1}^{N} p_{n}=P \\
\boldsymbol{H}=\left(h_{m n}\left\langle\boldsymbol{\omega}_{m}, \boldsymbol{a}_{m n}\right\rangle\right)_{1 \leq m \leq M, 1 \leq n \leq N}
\end{gathered}
$$

where the superscript $\mathrm{H}$ denotes the complex-conjugate transpose of a vector or matrix.

Let $\rho=P / \sigma^{2}, \lambda_{i}(i=1,2, \cdots, M)$ are the eigenvalues of the matrix $\boldsymbol{H H}^{\mathrm{H}}$.
Let $\tilde{\boldsymbol{H}}_{M \times N}=\left[\begin{array}{ccc}h_{11} & \cdots & h_{1 N} \\ \vdots & \ddots & \vdots \\ h_{M 1} & \cdots & h_{M N}\end{array}\right], \quad \tilde{\lambda}_{1}, \tilde{\lambda}_{2}, \cdots, \tilde{\lambda}_{M}$ are the eigenvalues of the matrix $\tilde{\boldsymbol{H}} \tilde{\boldsymbol{H}}^{\mathrm{H}}$.

Then we have following theorems.

Theorem 1. In the case that the DOAs from the all transmit antennas are the same in a receive antenna array, i.e. $\boldsymbol{a}_{m 1}=\boldsymbol{a}_{m 2}=\cdots=\boldsymbol{a}_{m N} \triangleq \boldsymbol{a}_{m}$, and the power is allocated to the transmit antennas equally, then the capacity of the SAMIMO system can be formulated as:

$$
C=\sum_{i=1}^{M} \log _{2}\left(1+\frac{\rho L}{N} \tilde{\lambda}_{i}\right)
$$

if and only if the optimal beam-forming vector can be written as

$$
\boldsymbol{\omega}_{i}^{*}=\frac{1}{\sqrt{L}} \boldsymbol{a}_{i}, \quad i=1,2, \cdots, M
$$

Proof: Since the DOAs of all elements of one smart antenna array are same, the channel matrix $\boldsymbol{H}$ can be written as

where

$$
\boldsymbol{H}=\tilde{\boldsymbol{W}} \tilde{\boldsymbol{H}}
$$

$$
\tilde{\boldsymbol{W}}_{M \times M}=\left[\begin{array}{ccc}
\boldsymbol{\omega}_{1}^{H} \boldsymbol{a}_{1} & & 0 \\
& \ddots & \\
0 & & \boldsymbol{\omega}_{M}^{H} \boldsymbol{a}_{M}
\end{array}\right],
$$

In addition that equal transmit power allocation, the capacity is given by

$$
\begin{gathered}
C(\boldsymbol{\omega})=\max _{\boldsymbol{\omega}}\left\{\log _{2} \operatorname{det}\left(\frac{\rho}{N} \tilde{\boldsymbol{W}} \tilde{\boldsymbol{H}} \tilde{\boldsymbol{H}}^{\mathrm{H}}(\tilde{\boldsymbol{W}})^{\mathrm{H}}+\boldsymbol{I}_{M}\right)\right\} \\
\text { s.t. } \\
\left\|\boldsymbol{\omega}_{m}\right\|=1, m=1,2, \cdots, M
\end{gathered}
$$

Using singular value decomposition (SVD), we can write

$$
\tilde{\boldsymbol{H}} \tilde{\boldsymbol{H}}^{\mathrm{H}}=\tilde{\boldsymbol{U}}^{\mathrm{H}} \tilde{\Lambda} \tilde{\boldsymbol{U}}
$$

where $\tilde{U}$ is a unitary matrix, and $\tilde{\Lambda}$ is a non-negative diagonal matrix whose diagonal elements are the singular values of the matrix $\tilde{\boldsymbol{H}} \tilde{\boldsymbol{H}}^{\mathrm{H}}$,i.e., $\tilde{\Lambda}=\operatorname{diag}\left(\tilde{\lambda}_{1}, \tilde{\lambda}_{2}, \cdots, \tilde{\lambda}_{M}\right)$, so we obtain

$$
\begin{aligned}
& \log _{2} \operatorname{det}\left(\frac{\rho}{N} \tilde{\boldsymbol{W}} \tilde{\boldsymbol{H}} \tilde{\boldsymbol{H}}^{\mathrm{H}} \tilde{\boldsymbol{W}}^{\mathrm{H}}+\boldsymbol{I}_{M}\right) \\
& =\log _{2} \operatorname{det}\left(\frac{\rho}{N} \tilde{\boldsymbol{W}} \tilde{\boldsymbol{U}}^{\mathrm{H}} \tilde{\Lambda} \tilde{\boldsymbol{U}} \tilde{\boldsymbol{W}}^{\mathrm{H}}+\boldsymbol{I}_{M}\right) \\
& =\log _{2} \operatorname{det}\left(\frac{\rho}{N} \tilde{\Lambda} \tilde{\boldsymbol{U}} \tilde{\boldsymbol{W}}^{\mathrm{H}} \tilde{\boldsymbol{W}} \tilde{\boldsymbol{U}}^{\mathrm{H}}+\boldsymbol{I}_{M}\right)
\end{aligned}
$$

To simplify the rest of the derivations, let 


$$
\boldsymbol{A}=\tilde{\boldsymbol{U}} \tilde{\boldsymbol{W}}^{\mathrm{H}} \tilde{\boldsymbol{W}} \tilde{\boldsymbol{U}}^{\mathrm{H}}, \boldsymbol{B}=\frac{\rho}{N} \tilde{\Lambda} \boldsymbol{A}+\boldsymbol{I}_{M}
$$

Since $\boldsymbol{B}$ is positive definite Hermitian matrix, from Hadamard theorem [9], we obtain

$$
\begin{array}{r}
\log _{2} \operatorname{det}\left(\frac{\rho}{N} \tilde{\Lambda} \tilde{\boldsymbol{U}} \tilde{\boldsymbol{W}}^{\mathrm{H}} \tilde{\boldsymbol{W}} \tilde{\boldsymbol{U}}^{\mathrm{H}}+\boldsymbol{I}_{M}\right) \\
\leqslant \log _{2} \prod_{i=1}^{M} \boldsymbol{B}_{i i}=\log _{2} \prod_{i=1}^{M}\left(1+\tilde{\lambda}_{i} \frac{\rho}{N} \boldsymbol{A}_{i i}\right)
\end{array}
$$

here $\boldsymbol{A}_{i i}$ and $B_{i i}$ are diagonal elements of $\boldsymbol{A}$ and $\boldsymbol{B}$, respectively. In (10), there can be equality only if $\boldsymbol{B}$ is a diagonal matrix. If $\boldsymbol{A}$ is a diagonal matrix, $\boldsymbol{B}$ is a diagonal matrix. So equation (10) is established only when $\boldsymbol{A}$ is a diagonal matrix. According the expression of $\boldsymbol{A}$, it can be known that all diagonal elements are independent

$$
\begin{aligned}
& \boldsymbol{A}_{i i}=\sum_{j=1}^{M}\left|\boldsymbol{\omega}_{j}^{\mathrm{H}} \boldsymbol{a}_{j}\right|^{2}\left|u_{i j}\right|^{2} \\
& \leqslant \sum_{j=1}^{M}\left\|\boldsymbol{\omega}_{j}^{\mathrm{H}}\right\|^{2}\left\|\boldsymbol{a}_{j}\right\|^{2}\left|u_{i j}\right|^{2}=L \sum_{j=1}^{M}\left|u_{i j}\right|^{2}=L
\end{aligned}
$$

In (11), there can be equality if and only if $\omega_{i}$ is given by

$$
\boldsymbol{\omega}_{i}^{*}=\frac{1}{\sqrt{L}} \boldsymbol{a}_{i}, \quad i=1,2, \cdots, M
$$

and after substitution, the capacity can be written as

$$
C=\sum_{i=1}^{M} \log _{2}\left(1+\frac{\rho}{N} \tilde{\lambda}_{i} L\right)
$$

if and only if the beam-forming vector is given by (12).

Theorem 2. In the case that the DOAs from different tranmit antennas are not the same in a receive antenna array, the upper bound of the capacity of the SA-MIMO system with equal power can be written as

$$
\begin{aligned}
& C=\log _{2} \prod_{i=1}^{R}\left(1+\frac{\rho}{N} \lambda_{i}\right) \\
& \leqslant R \log _{2}\left(1+\frac{\rho}{R N} \sum_{m=1}^{M} \lambda_{m, \max }\right)
\end{aligned}
$$

where $R$ is the number of singular values of matrix $\boldsymbol{H} \boldsymbol{H}^{\mathrm{H}}$.

Proof: In the case of equal transmit power allocation, we can obtain the equivalent problem

$$
\begin{gathered}
C(\boldsymbol{\omega})=\max _{w}\left\{\log _{2} \operatorname{det}\left(\frac{\rho}{N} \boldsymbol{H} \boldsymbol{H}^{\mathrm{H}}+\boldsymbol{I}_{M}\right)\right\} \\
\text { s.t. } \\
\left\|\boldsymbol{\omega}_{m}\right\|_{\mathrm{F}}=1, m=1,2, \cdots, M
\end{gathered}
$$

Using SVD, we can write

$$
\boldsymbol{H} \boldsymbol{H}^{\mathrm{H}}=\boldsymbol{U}^{\mathrm{H}} \Lambda \boldsymbol{U}
$$

where $\boldsymbol{U}$ is a unitary matrix, $\Lambda=\operatorname{diag}\left(\lambda_{1}, \lambda_{2}, \cdots, \lambda_{R}, 0,0 \cdots 0\right)$

$$
\lambda_{1} \geq \lambda_{2} \geq \cdots \geq \lambda_{R}, \sum_{i=1}^{R} \lambda_{i}=\operatorname{Tr}\left(\boldsymbol{H} \boldsymbol{H}^{\mathrm{H}}\right) .
$$

So

$$
\log _{2} \operatorname{det}\left(\frac{\rho}{N} \boldsymbol{H} \boldsymbol{H}^{\mathrm{H}}+\boldsymbol{I}_{M}\right)=\log _{2} \prod_{i=1}^{R}\left(1+\frac{\rho}{N} \lambda_{i}\right)
$$

From the theorem of the arithmetic and geometric means, we obtain

$$
\begin{aligned}
\log _{2} \prod_{i=1}^{R}\left(1+\frac{\rho}{N} \lambda_{i}\right) & \leqslant \log _{2}\left(\sum_{i=1}^{R}\left(1+\frac{\rho}{N} \lambda_{i}\right) / R\right)^{R} \\
& =R \log _{2}\left(1+\frac{\rho}{R N} \sum_{i=1}^{R} \lambda_{i}\right)
\end{aligned}
$$

with equality when

$$
\lambda_{1}=\lambda_{2}=\cdots=\lambda_{R}
$$

From Rayleigh-Ritz theorem [9], we obtain

$$
\begin{aligned}
& \sum_{i=1}^{R} \lambda_{i}=\operatorname{Tr}\left(\boldsymbol{H} \boldsymbol{H}^{\mathrm{H}}\right)=\|\boldsymbol{H}\|^{2} \\
& =\sum_{m=1}^{M} \boldsymbol{\omega}_{m}^{\mathrm{H}}\left(\sum_{n=1}^{N}\left|h_{m n}\right|^{2} \boldsymbol{a}_{m n} \boldsymbol{a}_{m n}^{\mathrm{H}}\right) \boldsymbol{\omega}_{m} \leqslant \sum_{m=1}^{M} \lambda_{m, \max }
\end{aligned}
$$

In (19), there can be equality when $\boldsymbol{\omega}_{m}$ is main-eigenvector of $\boldsymbol{A}_{m}$, where

$$
\boldsymbol{A}_{m}=\sum_{n=1}^{N}\left|h_{m n}\right|^{2} \boldsymbol{a}_{m n} \boldsymbol{a}_{m n}^{\mathrm{H}}
$$

Namely,

$$
\begin{aligned}
& \boldsymbol{A}_{m} \boldsymbol{\omega}_{m}=\lambda_{m, \max } \boldsymbol{\omega}_{m}, \\
& \left\|\boldsymbol{\omega}_{m}\right\|=1, \quad \forall m=1,2, \cdots, M
\end{aligned}
$$

So the upper bound of the capacity of the SA-MIMO system with equal power can be written as 


$$
\begin{aligned}
& C=\log _{2} \prod_{i=1}^{R}\left(1+\frac{\rho}{N} \lambda_{i}\right) \\
& \leqslant R \log _{2}\left(1+\frac{\rho}{R N} \sum_{i=1}^{R} \lambda_{i}\right) \\
& \leqslant R \log _{2}\left(1+\frac{\rho}{R N} \sum_{m=1}^{M} \lambda_{m, \max }\right)
\end{aligned}
$$

with equality when equation (18) and equation (21) are true.

\section{NUMERICAL RESULTS}

Monte Carlo simulations are carried out under some cases to compare the capacities of SA-MIMO and conventional MIMO systems. We denote a SA-MIMO system with $N$ transmit antennas and $M$ receive antenna arrays and each array owning $L$ elements by $N \times(M, L)$ SA-MIMO.

Fig. 2 depicts the ergodic channel capacity of MIMO and SA-MIMO for various values of signal-to-noise ratio (SNR). It is shown that smart antennas can bring significant capacity gain for the MIMO system without additional spatial degrees of freedom. It is also evidenced that the upper bound is actually very tight for the system considered.

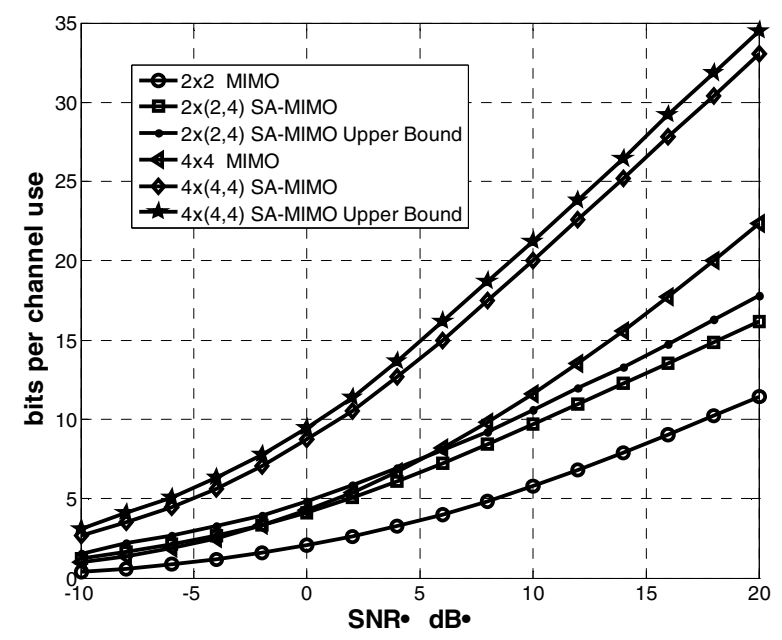

Fig. 2 Ergodic Capacity Comparison between MIMO and SA-MIMO

The following simulations are performance under the assumption that the transmit power are equally allocated and the DOAs from different transmit antennas are the same at the same receive antenna array.

In Fig. 3, we plot the capacity of a deterministic MIMO channel for different numbers of smart antenna array elements at receiver at different SNRs. Assume that there are two transmit and two receive arrays, and the channel matrix is given by

$$
\tilde{\boldsymbol{H}}_{2 \times 2}=\left[\begin{array}{lr}
-1.408-0.4462 \mathrm{i} & 1.0664+0.3809 \mathrm{i} \\
0.8241-0.2013 \mathrm{i} & 1.5575+0.7058 \mathrm{i}
\end{array}\right]
$$

It is shown that the capacity increases as the number of smart antenna array element does.

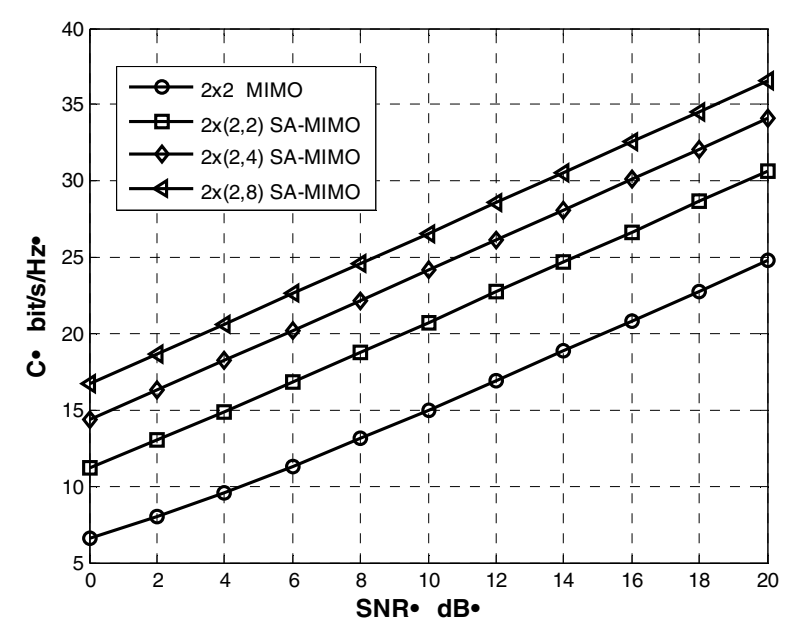

Fig. 3 capacity for different numbers of smart antenna array elements

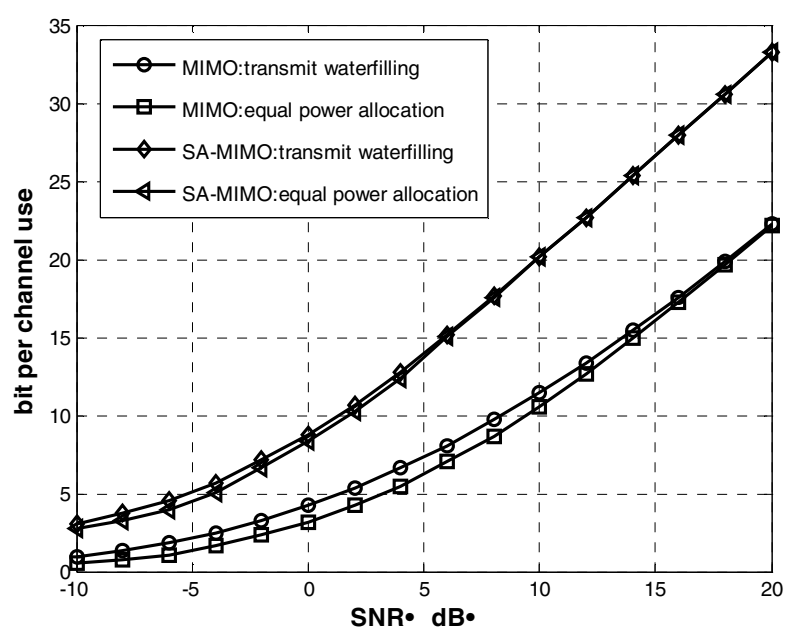

Fig. 4 Ergodic Capacity Comparison for defferent power allocation

Fig. 4 depicts the ergodic channel capacities of MIMO and SA-MIMO with optimal power allocation and equal power allocation under the case that $N=4, M=4, L=4$, respectively. We observe that optimal power allocation performs better than equal power allocation in MIMO system. However the gap of the performance between optimal power allocation and equal power allocation is small because smart antenna improves the channel and SNR in SA-MIMO system. So substitution equal power allocation for optimal power allocation in SA-MIMO system can reduce system complexity for commercial wireless applications.

\section{CONCLUSIONS}

In the case of the same DOAs from different transmit antennas at the same receive antenna array, we have deduced the closed-form expression of SA-MIMO system with equal power allocation. In the case of the different DOAs, an upper bound of the capacity with equal power allocation was derived.

Forthcoming work should be focused on analyzing the capacity gain of SA-MIMO systems under the multiple users 
case in which the advantage suppressing interference among users can embody.

\section{ACKNOWLEDGMENT}

This work is supported by National Science and Technology Key Special Projects (No. 2010ZX03003-002 \& 2010ZX03003-004), National Nature Science Foundation of China (No. 60972023), and Research Fund of National Mobile Communications Research Laboratory, Southeast University (No. 2011A06), and also supported by UK-China Science Bridge : R\&D of (B)4G Wireless Communications System.

\section{REFERENCES}

[1] E. Telatar, "Capacity of multi-antenna Gaussian channels," Eur. Trans. Telecommun., vol. 10, no. 6, Nov.-Dec. 1999,pp. 585-595.

[2] J. H. Winters, J. Salz, and R. D. Gitlin, "The Impact of Antenna Diversity on the Capacity of Wireless Communication Systems," IEEE Transactions on Communications, COM-42, February/ March/April 1994, pp. 1740-51.
[3] T. L. Marzetta and B. M. Hochwald, "Capacity of a mobile multiple antenna communication link in Rayleigh fading," IEEE Trans. Inform. Theory, vol. 45, pp. 139-158, Jan. 1999.

[4] Lizhong Zheng; Tse, "Diversity and Multiplexing: A Fundamental Trade-Off in Multiple-Antenna Channels," IEEE Trans. on D.N.C. Information Theory, Vol. 49, No. 5, May 2003, pp. 1073-1096.

[5] Andersen, J. B., "Array Gain and Capacity for Known Random Channels with Multiple Element Arrays at Both Ends," IEEE J. Select. Areas Commun., Vol. 18, November 2000, pp. 2172-2178.

[6] Pedersen, K. I., and P. E. Mogensen, "Performance of WCDMA HSDPA in a Beamforming Environment Under Code Constraints," IEEE 58th Vehicular Technology Conference, Vol. 2, October 6-9, 2003, pp. 995-999.

[7] Ma You, ZHENG Zhi, ZHOU Yu-long, "Study Incorporation of Smart Antennas into MIMO System"[J], Communications Technology, Vol.43 , No.05, 2010, pp.173-175.

[8] Zhu, F., Lim, M.S, "Combined beamforming with space-time block coding using double antenna array group” [J]. ELECTRONICS LETTERS 24th June 2004 Vol. 40 No. 13, pp.811-813.

[9] Bellmam R. "Introduction to Matrix Analysis"[M],Mcgraw-Hillbook company, 1970. 